\title{
Industrial biopolymers
}

\section{from Paul Calvert}

THE term biopolymers really includes al macromolecules of biological origin but, for industrial application, biopolymers can be divided into two categories: those to be used in aqueous solution and those to be used as solids.

The first category contains a wide range of polysaccharide thickeners, emulsifiers and gelling agents used in the food industry and in a wide range of printing and coating applications. These materials can be extracted from trees, seeds and seaweeds or can be manufactured by modifying starch or cellulose. A variety of microorganisms also produce water soluble extracellular polysaccharides and it was a microbial product - Xanthan gum - that was the centre of attention at a recent meeting in Birmingham*. Xantham gum is produced commercially by Kelco from Xanthomonas campestris and consists of a glucose main chain with three-unit side chains (mannose-glucoronic acidmannose) on every other glucose residue. It was picked for its unusual gelling properties from a survey of microbial polysaccharides carried out by Allene Jeanes at the US Department of Agriculture in the 1950s. It is pseudoplastic, that is it forms a gel or high viscosity solution which thins when the solution is stirred. The viscosity is stable to changes in temperature, $\mathrm{pH}$ and salt concentration but can be modified by adding other vegetable gums. This gives it a wide range of uses, for example in 'instant' desserts and in salad dressings that flow out of the bottle but not off the salad.

One very important possible application for water soluble polymers is in the secondary recovery of oil. A normal oil well only produces about 40 per cent of its oil content in the combined processes of primary recovery, where the oil comes out under its own pressure, and secondary recovery where it is forced out by water or gas pumped down a series of holes surrounding the oil reservoir. The extraction is limited by the failure of the water to displace oil from porous rock and by the tendency of the water to follow cracks in the rock directly to the central well rather than sweeping the oil before it. There are a variety of ways to enhance oil recovery (for a review see Arnold, C.W. in Microbial Polysaccharides and Polysaccharases ed. Berkeley, Gooday, \& Ellwood, SGM/Academic Press, 1979) but a possible new approach is through a combination of surfactants, to allow the water to displace the oil from rock, with water-soluble polymers to increase the water viscosity and prevent it by-passing the oil. The requirements for such a polymer are that it gives a high viscosity increase at low concentrations but is shearthinning for ease of pumping. It should

Paul Calvert is in the School of Molecular Sciences, University of Sussex.

also be stable at the bottom of an oil well for several months, which, in the case of deep North Sea wells where conditions are most severe, means it must be stable in saline at temperatures of about $120^{\circ} \mathrm{C}$. Polyacrylamides have been used successfuly in the US although the cost was high compared to the value of the oil produced. For general use, polyacrylamides are too unstable and too easily absorbed and every known water soluble polymer has been considered as an alternative. Xanthan gum or a similar microbial polysaccharide seem to be suitable but current fermentation methods of production make them expensive. Also, at a rate of one pound of polymer used per barrel of oil produced there may not be sufficient production capacity for the polysaccharides to satisfy the potential demand even if all fermentation plants were employed for this one product.

In the light of the increasing price of oil and the current interest in fermentation methods of producing industrial chemicals it seems natural to seek an industrial plastic made by microorganisms. For a biopolymer to be used as a plastic it should be a

"A symposium on "Recent advances and Industrial Chemical Engineers was held in Birmingham in March

\section{Essential fatty acids and prostaglandins}

\section{from Michael Crawford}

IN 1930 at the University of Minnesota in Minneapolis, George and Mildred Burr made the remarkable discovery that dietary linoleic acid was essential for growth, development and normal function in rats. At this time, pharmacological activity of a group of uncharacterised substances from semen and male accessory glands was also being studied by U.S. von Euler at the Karolinska Institute in Stockholm. He characterized the material as a biologically active hydroxylated unsaturated fatty acid and named it 'prostaglandin'.

World War II interrupted research on the 'essential fatty acids' (EFA) and 'prostaglandins' (PG), and it was not until the 1950s, with the introduction of radioisotopes, gas-liquid chromatography and mass spectrometry that further progress was made. During the 1950s and 1960s EFAs were shown to be required by several species, including man, and linoleic acid was found to be metabolized to longer chain polyunsaturated acids. In 1964 these were established as the precursors for the PGs by Van Dorp and colleagues of Unilever in Holland and by Bergstrom and colleagues at the Karolinska Institute in Sweden.

In spite of this evidence establishing a Michael Crawford is in the Nuffield Laboratories, London and was one of the Congress program coordinators. linear uncross-linked molecule insoluble in hot water but soluble in organic solvents or, better, capable of melting and processing without decomposition. Given that natural structural polymers like cellulose and collagen tend to be made from polar monomers and derive their insolubility from strong intermolecular hydrogen bonding it is not surprising that suitable examples are rare. One example is poly ( $\beta$-hydroxybutyrate) which is an optically active thermoplastic polyester with a melting point around $175^{\circ} \mathrm{C}$ produced as an intracellular storage compound by various bacteria inlcuding Azotobacter vinelandii and Pseudomonas solanasearum.

If a suitable naturally produced thermoplastic were available it may still not be practicable to manufacture it. Any fermentation process leads to products which must be separated and purified and this may be considerably more expensive than a simple catalytic polymerisation, (for example, ethylene to polyethylene) although it may be competitive with the more expensive condensation processes leading to nylons or polyethylene terephthalate.

Thus while the traditional biopolymers, cellulose and rubber, are still the most important and water soluble biopolymers are in production, a solid polymer, the plastic microbe, has yet to come. $\square$ link between EFA and the PGs, work in both fields remained separate. Pharmaceutical companies became excited by the potential of PGs, their analogues and inhibitors in the fields of reproductive physiology, inflammation and thrombosis. Meanwhile in the EFA camp, advances were made in establishing the role of EFA in cell membrane function, brain growth and the prevention of atherosclerosis and thrombosis. At the time, however, benefits of high linoleic acid diets to the cardiovascular system were largely attributed to reduction in blood cholesterol. The important role of EFAs and PGs may well be in the integrity of vascular membranes, the stability of platelets and in modulation of vascular tone.

A recent Congress* was held to bring the two fields together. Both Burr and von Euler attended the Congress as guests of honour. The sessions were designed to highlight the precursor-prouluct relationship between EFAs, PGs and other biologically important EFA metabolites.

A number of different speakers independently supported the idea that nutritional management of dietary precursors should affect biosynthesis of PG and other oxygenated products. The practical applications of this include the reduction

*The Golden Jubilee Congress on Essential Fatty Acids and Prostaglandins was held at the University of Minnesota, May 4-7, 1980. 